\title{
Inclusive game design facilitating shared gaming experience
}

\author{
UIf Wilhelmsson ${ }^{1} \cdot$ Henrik Engström ${ }^{1}$ • \\ Jenny Brusk ${ }^{1} \cdot$ Per Anders Östblad ${ }^{1}$
}

Published online: 5 April 2017

(C) The Author(s) 2017. This article is an open access publication

\begin{abstract}
This article presents the result from a study comparing the perception and understanding of a game story between sighted and visually impaired players playing the same game. In particular, whether sighted and visually impaired players could experience and recount the same story construed from the plot elements that are either manifested by audio and graphics in the case of sighted players or primarily by audio in the case of visually impaired players. To this end, we have developed a graphical point-and-click adventure game for iOS and Android devices that aims to show how inclusive game design may be used to facilitate a shared gaming experience between sighted and visually impaired players. The game provides players with audio feedback that enables visually impaired players to interact with and experience the game, but in a manner that does not interfere with the overall appearance and functionality of the game. Thus, it has been designed to be fully inclusive to both groups of players and to give the same gaming experience when it comes to story content. The game has been evaluated through formal user tests where subjects have been asked to play the first chapter of the game followed by an interview. The study shows that the perception of the story was almost identical between the two groups. Generally it took visually impaired players a little longer to play the game but they also seem to listen more carefully to the dialogue and hence also build a slightly deeper understanding of the characters. The study also shows that the sighted players did not respond negatively towards the inclusive game design employed in the game.
\end{abstract}

Keywords Inclusive game design · Narrative · Story · Plot elements · Visually impaired players $\cdot$ Sighted players

Henrik Engström

henrik.engstrom@his.se

1 School of Informatics, University of Skövde, PO Box 408, 54128 Skövde, Sweden 


\section{Introduction}

Play and games have probably always been key elements of culture and as such have played a pivotal role for the development of civilization (Caillois 1961/2001) A reason for this is that play and games have characteristic functions of social inclusion, making it possible for diverse groups of people to share a collective experience through the act of playing the same kind of games within a culture. As such, play is a means for social interaction that transcends age groups and it is not uncommon that family and friends of different ages choose to gather around a game when they want to engage in a joint social activity. Over the last decade computer games in particular have become more widespread and popular among the population as a whole, independently of age and gender; today "everyone" plays video games (see for example Entertainment Software Association 2015; Media Council 2013, 2015; Ipsos MediaCT and The Interactive Software Federation of Europe 2012). This is also reflected in the Swedish video game industry, which has shown a substantial growth in economical turnover over the last years (Dataspelsbranschen 2015).

Video games show a variety concerning, for example, theme, aesthetics, graphical content, and sound design. They can be played on various platforms spanning from arcade cabinets to computers to consoles to mobile devices including smartphones. The number of children in Sweden having access to various platforms for playing video games has increased substantially during this period (Swedish Media Council 2013, 2015). For example, the access to tablets amongst 9 year olds in Sweden almost quadrupled between 2012/2013 and 2014/2015, from 12 to $42 \%$. The amount of younger children owning smartphones has also increased; $78 \%$ of the 9-12 and 98\% of all 13-19 year olds own a smartphone (Swedish Media Council 2015). Furthermore, Swedish video game industry statistics shows that $43 \%$ of the games are distributed on mobile platforms and that the game industry overall has become less platform bound than it used to be (Dataspelsbranschen 2015). According to the Swedish Media Council (2015), almost all of Swedish children ages 9-12 play video games (98\% of the boys and $97 \%$ of the girls). While boys continue to play in their teenage, girls tend to favour social media instead. The amount of time spent on playing video games also differs substantially between these groups. While less than $10 \%$ of the girls play $3 \mathrm{~h}$ or more per day, almost every other teenage boy play at least $3 \mathrm{~h}$ per day. The total amount of children spending $3 \mathrm{~h}$ or more playing video games on tablets has increased amongst the 10-11 year olds and the 13-14 year olds compared to the prior study (Swedish Media Council 2015). The prior study (Swedish Media Council 2013) reports that while girls prefer to play solo, boys in this age group tend to play games in a social context with friends, siblings, and to a lesser degree, parents or other grown-ups and that the percentage of players playing video games in solitude has decreased. What all these numbers show, is that video games are to be understood as one of the more important leisure activities for large groups of children. However, there are children that are physically or socially excluded from this increasingly important social context. Not because they don't own or have access to a smartphone or tablet, but 
because they are visually impaired to a higher or lesser degree and therefore cannot experience the majority of the commercially available games on equal terms with sighted players. Only quite recently, well-functioning support systems have been built into the operating system on computers running a graphical user interface or into mobile units such as smartphones or tablets to make these more accessible. Apple's iOS VoiceOver system is an example hereof.

The vast majority of mainstream commercially available video games rely heavily on their graphical components as the main communication with the player and is used for defining and diversifying game characters, creating the game environment, and separating interactable objects from non-interactable objects. Even the term video game in itself is part of a visually dominated culture. The digital graphics of video games have evolved substantially since the 1980s and today the smartphones and tablets completely outrun the game consoles of yesteryear by far when it comes to technological advances and specifications such as image resolution, colour depth, and asymmetric composition of the game environment. That is to say that a lot of effort has been made in order to increase the technical capabilities as well as the artistic qualities of the graphical components of video games on all distribution platforms. Of course there has also been technological development of game audio, such as higher sample rate, increased bit depth, the possibility to use pre-recorded sounds rather than chip generated sounds, built in audio effect processing such as reverberation, multiple audio channels, and multiple speakers. This have made new sound aesthetics in video games possible. These advances have thus for the most part been used to sustain and enhance a sighted player's experience of a game, and to a much lesser degree to make video games more accessible to visually impaired players.

This development has led to that very few of the games on the market are accessible to visually impaired players and those that are, are usually designed primarily for the visually impaired, rendering them less attractive for players that are not (Atkinson et al. 2006; Ekman 2007; Grammenos et al. 2009). One of the reasons is possibly that the economical incitements for producing commercial video games primarily for visually impaired players are small. According to the national organisation for the visually impaired in Sweden (Synskadades riksförbund 2016a), the total number of visually impaired persons in Sweden is approximately 120,000, of which around 3000 are children. Hence this is not a mass market, why it seems that a majority of video game companies do not address this subject at all or to a very small extent. Such an effort would less likely have any huge impact on the economical turnover for the industry at large or even for specific game studios. On the other hand, for the individual that is excluded there would be much to gain by being part of game culture. Also, a more inclusive approach works in the opposite direction as well and invites the sighted players to play the very same games as visually impaired players.

Organizations, such as the AbleGamers Foundation, have had a positive effect on some of the larger game developers to make more inclusive game designs concerning different kinds of impairments.

This lack of inclusive games creates inaccessibility to a progressively important social context-game culture-for the visually impaired. There is a risk that an 
already marginalized and to some extent socially excluded group will be even more marginalized if they are not given the same possibilities and opportunities to participate in this particular social context and share the same kind of experiences on as equal terms as possible. We find this problematic, especially concerning visually impaired children since this constitutes an obstacle for them to play games, in the broad sense of the concept of games, and hence to gain access to the mediated culture communicated through the act of playing games of different kinds.

In an attempt to bridge this gap, we have developed a graphical point-and-click adventure game called Frequency Missing for iOS and Android devices. The game has been designed to provide players with audio feedback that enables visually impaired players to interact with and experience the game, but in a manner that does not interfere with the overall appearance and functionality of the game. This is a design that is fully inclusive to both groups of players and that is as invisible for sighted players as possible without hindering visually impaired players to share the same gaming experience when it comes to story content.

\section{Background}

It is quite usual to share ones experiences with others in a social context such as playing games. Play and games possess a social dimension in that even solitary play and games often turn into competitive play between potential players (Caillois 1961/ 2001). This can manifest itself by players comparing individual skills such as high scores, chat about their experiences and progress in games. In other words, play and game, even though competitive or played solitary, are part of an important sociocultural context. Games are vehicles of social and cultural content and there is a constant interplay between games on the one hand and society/culture on the other. Furthermore, "Play is not merely an individual pastime. It may not even be that as frequently as is supposed." (Caillois 1961/2001, p. 37).

Video games are in respect to this not all that different from other types of games and they have rapidly become a large part of everyday life for children (Swedish Media Council 2015) as well as adults (Entertainment Software Association 2015).

As Embrick et al. (2012, p. Xv) notes "There is a tendency to dismiss online virtual programs such as World of Warcraft and Everquest as 'games' that people play either for fun, or to escape the realities of everyday life. What many of us fail to recognize is that these communities, although virtual, are extensions of the society in which they reside." Video games replicate social structures outside of games and to be accepted as a gamer on equal terms within a community of gamers requires a player that is able to enter the social context of game playing. This might be problematic for visually impaired gamers.

There has been a trend to integrate visually impaired and sighted pupils in the same educational situations during the last three decades (Stukat 2002). In Sweden, prior to 1986, visually impaired children without other disabilities mostly attended the special boarding school for the blind, Tomteboda, but since 30 years they are part of the ordinary school system and hence normally have classmates that are not visually impaired. The main reason for this was to promote social inclusion and 
reduce marginalization (Synskadades riksförbund 2016b). Special education for different groups of pupils does not always lead to good results as far as integration goes. As has been pointed out by Söderqvist Dunkers (2011) this attempt to include visually impaired children was not unproblematic. There are thresholds that need to be overcome in the social relations in the school environment's peer culture. According to Svensson referred to by Söderqvist Dunkers (2011) only one third of the visually impaired pupils had good social relations with other pupils in their initial years in ordinary schools. The same is valid for games and play. Special games and play designed to address specific disabilities at the cost of overall game design is not inclusive but separating even more. Play has in its essence a potential of integration. It could serve as a tool for inclusion. Corsaro (1997) notes that in order to successfully enter play in a peer culture, a player needs to first position him/ herself in a socially accepted distance to the players. The next step is to find out what the theme of the play is, what the content and scope of the play is and then make a useful contribution to the play activity that the peers playing can accept. For a blind player, who needs to rely on auditory information and touch, finding out what the theme is can be difficult in many play situations. Making a useful contribution is likewise problematic for the same reason.

Games targeted at visually impaired players are often not inclusive with respect to sighted players, or are designed to use only audio to communicate with the player which most sighted players are unfamiliar with (Atkinson et al. 2006; Ekman 2007; Grammenos et al. 2009; Oren 2007).

To completely rely on audio in game design is still uncommon and could be considered as a subculture in itself. Two rather well known and well-received games that are designed to use only audio to play the game are Papa Sangre (Somethin' Else 2010) and Papa Sangre II (Somethin' Else 2012). The first of these was awarded Most Innovative Game at Mobile Gaming Awards in 2011, and Papa Sangre II had a rating of $92 \%$ on the iOS platform in 2013, making it the bestreviewed game on $\mathrm{iOS}$ that year. But awards and positive ratings do not necessarily indicate that these two games have or will set a new standard for how games are primarily designed. They are novelties and very interesting games, but they have not yet had too much impact on the video game industry at large.

\section{Point and click adventure/puzzle games as a point of departure}

Adams (2010) defines adventure games as games that incorporate an interactive story in which the player plays the protagonist. These kinds of games are based on storytelling and the exploration of the game environment. A large part of the game play is based on solving puzzles and conceptual challenges. On the other hand there are few or no action challenges, no economic management or combats (Adams 2010). We chose this specific type of game since inherent in these games' basic structure is a reliance upon text and spoken words to communicate the key plot elements of the game. The first adventure games, such as Colossal Cave Adventure (a.k.a ADVENT) (CRL 1976) and Zork (Infocom 1980) proved this point as they relied solely on written language as a means to play the game. Games like these are 
story driven and rather linear. They start from the very beginning of the chain of events and the player follows that path to the end of the game (which might vary due to the choices the player makes during game play). Later games that combine graphical manifestations of the game environment with written language and spoken word, sound effects and music, have commonalities with the text only games: riddles, puzzles, problem solving and exploring the game environment. Such games, for example The Secret of Monkey Island (Lucas Arts 1990) and Day of the Tentacle (Lucas Arts 1993a), also established a structure how written language is displayed on the screen, how the player can control what to say in a dialogue with game characters, and how they can store objects they pick up along the path in an inventory. A game such as Day of the Tentacle is due to its basic design and organization of interactable dialogue on the screen, a good point of departure to construe a game that can be played without any graphical content as long as the structure of the interface is consistent throughout the game. As long as the player can correctly target and identify the interactable objects on the screen the game can be played.

Quite a few games in the genre of point and click adventure/puzzle games use a voice over from a first person point of being to communicate with the player when clicking objects on the screen. This approach mimics the narrative style of other media such as novels and film where this is quite common and well established style of narration. Not least in the film noir detective tradition. Used in a game, this approach provides yet another level of communication; it makes possible a prosodic emphasis on certain qualities of the object when playing the game with sound. In a written narrative, the emphasis could be signalled by utilizing different typographical styles (for example bold or italics) or providing the reader this kind of information by writing how a specific utterance is said. In point and click adventure games it is part of the genre conventions to let the playable character say things like "I can't use these things together!" (Lucas Arts 1993b) to help the player notice specific qualities of interactable objects.

We deliberately chose a story driven design since that allowed us to study how well the content of the game was understood by the players that tested the game. Narratives within the same genre are quite uniform due to genre conventions developed over time. A mystery narrative, for instance, usually contains the same kind of plot elements making up the story. In the further discussion some key terminology needs to be explained. When we say story in the context of this article we mean the recountable chain of causal events constructed from the experienced plot elements. The plot elements are the actual interactable events presented to the player over duration of time. As such, the story is the mental construction that players make from all the plot elements, and is furthermore subject to speculation on what might happen in the next series of events triggered by the players actions within the game environment. That is to say, that while playing, the player constantly construct, deconstruct, and reconstruct the story when choosing between the plot elements present. Due to this, recounting the story after a play session is affected by how many of the plot elements that have been part of the interaction with the game and also, but to a lesser degree in our game, in which order the plot 
elements have been exposed to the player. This is not unique to games but is equally valid in how an audience interpret a story from narratives at large.

\section{Designing the game}

Frequency Missing is a rather linear, story-driven, mystery point and click adventure/puzzle game designed for iOS and Android tablets and smartphones. These are the dominating platforms among visually impaired as well as sighted users. It was therefore something that from the very outset connected visually impaired and sighted people, a common base level of using the same kind of devices. It also made possible a design that is touch based - the way to interact with objects on the screen is to touch them directly why there is no need to use a keyboard or mouse to play the game. Since we designed the game for sighted and visually impaired players we wanted as few input devices as possible as well as few interaction modes. This was to avoid the need for complicated tutorials and instructions, which could have made user testing unnecessarily complex.

The story, plot elements, characters, and setting are inspired by characteristic features of film noir but are set in the 1960's (rather than the typical 1940's settings of noir films). The game design makes use of false clues (so called red herrings). The plot elements are sometimes deliberately misleading, causing the player to make false assumptions on what will happen (or what has happened). The time period is manifested in the types of objects that can be found in the game environment and their visual design (see Fig. 1), as well as in the dark sounding jazz music played in the game. The music is performed in the format of a typical jazz noir ensemble. The main motivation for using this particular setting and era was that it opened up the possibility for us to use older indexical sounds like bell telephones

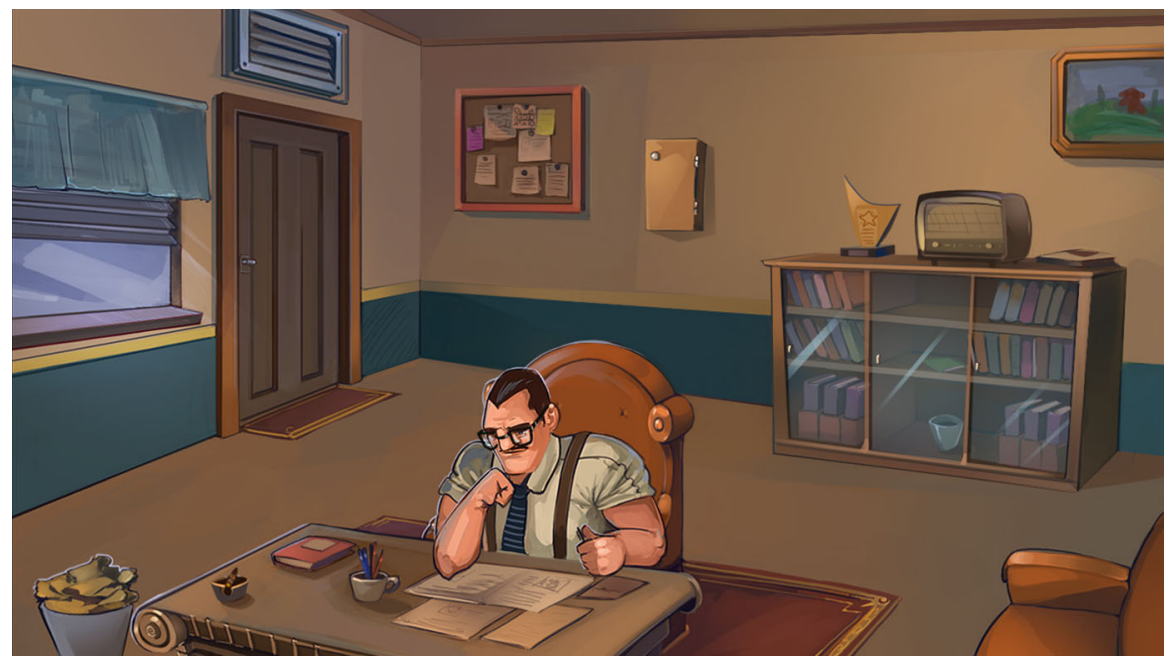

Fig. 1 A screen capture from Frequency Missing, illustrating its visual style 
and typewriters instead of their contemporary counterparts; mobile phones and computers. This choice made possible a distinct and diversified sound design where objects that produce sound clearly point to the presence of a specific object and sounds that stand out from one another. The sounds used to designate the presence of a telephone is for instance easily discernible from a sound designating a typewriter by virtue of the way their sounds would be actually produced in the real world outside the game. This was of importance since we wanted the sound of objects to be part of the navigation within the environment. The indexical sound design helps the player to find the objects' position relative to other objects. This together with unique ambiences and room-tones helps the player to get to know the different environments and makes it easier to recognize different rooms and places.

The game is centred entirely on a female reporter named Patricia. She is the focalizer (Genette 1983) of the events why it is from her presence in the game environment alone that the plot elements are possible to engage in. Patricia is the player's agent, the game ego, but she is not a visible character since the game is enacted from a mix of first person and God's perspective in its graphical construction. This design choice was made based on the hypothesis that having a character that has to walk around on screen could be a distraction, without adding any value to the audio-only players. Parts of the game are shown from a visual perspective, a point of observation, which would be impossible for Patricia to occupy. From a purely auditory position however, playing the game without seeing the graphics, this mix of observation points between locations in the game, does not affect game play.

There are no other playable characters in the game apart from Patricia, but quite a few non-playable characters that Patricia can interact with, mainly through dialogue organized in a branching tree structure. For sighted players the dialogue options are written on the screen and Patricia's inner voiceover is subtitled below the actual game environment, much like a movie. By playing Patricia, the player can also trigger reactions from the other characters by using some of the interactable objects in the game environment.

The game begins with a puzzle that aims to introduce the player to the interaction model. The player finds Patricia trapped in a secluded dark environment and the goal is to set her free. To do this, the player must explore the environment by dragging a finger over the screen searching for a way to get out of the secluded environment. By localising a series of screws and loosen them Patricia can be freed.

After having solved the first puzzle the next plot element is Patricia's first day at her new work at a radio station, which is preceded by a voice over and a text setting us back three days in time. The first puzzle is therefore chronologically one of the last parts of the game narrative. This strategy to kick-start the narrative is also quite typical in the film noir genre.

The player learns that Patricia's friend Richard, who helped her get the job at the radio station, has mysteriously disappeared. The objective of the game is to find out what happened to him. The setting of the remainder of the first chapter is the radio station; consisting of a lobby from which three offices and a newsroom are accessed. At the radio station, the player gets introduced to her new colleagues: Karl, a technician and Patricia's office mate; the manager (he is not given a name); Monica, 
the receptionist; and, Stephanie, the news anchor. Through dialogue with these characters, the player can find out more about the colleagues and the circumstances around Richard's disappearance. In addition to dialogue, the story is narrated by means of Patricia's inner voice, a common feature of point-and-click adventures.

A major challenge was to achieve clarity in the sound design and audio mix. Since the game must be equally playable independently of whether it is played with or without graphics, all crucial information and events that are conveyed by graphics must also be conveyed with sound to make the game playable. All rooms, for example, have unique ambiance tracks that reflect the setting of the scene. In the room depicted in Fig. 1, there is a low ventilation hum and a broadcast coming from the radio.

Similarly, all interactable objects have their own unique 3D sound, meaning the closer the pointing finger is to the object, the stronger the sound volume becomes, making it possible to tell whether the object is to the left or right of the finger. This in turn designates from which point in the environment the player perceives the sound. In addition to $3 \mathrm{D}$ sounds, when an interactable object is located, there is a clear and audible interface sound. If the player moves her finger onto the interactable object the interface sound is played normally and if the player moves her finger away from the interactable object the same sound is played in reverse. This design further helps the player to localize and pinpoint the exact location of any interactable object in the game. Every action and event that is not diegetic (directly connected to the game environment) is sonically enhanced and made protruding by an interface sound. All interface sounds are also separated from other sounds and made audible in the mix by being spread very wide in stereo width. This design further helps the player to separate the different types of sound from each other.

All dialogue is voice acted by experienced actors, following a hypothesis that using text-to-speech could reduce the level of immersion the player could experience. To make all speech clear and audible, since audio-only players can't access the subtitles, all background sounds (music, ambiance) are lowered in volume and filtered in their high frequencies whenever a character speaks.

At the basis of the sound design lies the combined model for computer game audio proposed by Wilhelmsson and Wallén (2011). See Fig. 3. This model allows for structuring the game audio with regards to the four basic categories Interface, Effect, Zone and Affect (IEZA) that are defined by Huiberts and van Tol (2008) and in accordance with their model whether the sounds are in-game sounds (diegetic) or not (non-diegetic) and whether they are player induced (activity) or not player induced (setting). In addition, the combined model also takes into account other qualities such as relative loudness of the sound objects and dominant frequency of a sound clip. In addition it provides an overview of how sounds in a particular part of a game are clustered with regard to cognitive load on the player. The latter part on cognitive load is based on the conceptual model proposed by Murch (1998), in which the layering of film sound is arranged in an arc spanning from embodied sounds to encoded sounds. Embodied sounds are sounds that do not demand the person that listen to the sound to intellectualize about the meaning of the sound. Murch uses music to designate this kind of sound. At the other end of the arc are the 
encoded sounds that do require an intellectual decoding process and an interpretation of the sound into something meaningful. Murch uses speech as an example hereof. In the combined model, sounds that are embodied are placed at the outer part of a circle and sounds that are encoded are placed closer to the centre. The reason for this is that it rapidly visualizes the amount of encoded sounds that could overload the player.

Figures 2 and 3 show the same part of the game, Karl's office. Figure 2 is a concept art drawing showing the distribution of the interactable sound objects on the screen marked by blue circles. Figure 3 is the clustering of the clips using the combined model. The latter figure shows the relative loudness of the sound objects, their dominant frequency, their category and the possible cognitive load on the player. The closer to the centre of the circle a sound is located, the more encoded it is. In Fig. 3 the sounds 9 and 10, are dialogue sequences and hence considered to be effect sounds. Their dominant frequencies are within the midrange of the sound spectrum why they are designated by squares. At the other extreme, sound 4 is located at the outer part of the circle, designated by a small circle since its dominant frequencies are within the bass spectrum and it is less loud than sounds 9 and 10.

The visual design of the environment was construed backwards compared to more traditional ways. The game designer and audio designer first made a map of where the interactable objects should be placed. This was made to avoid that the $3 \mathrm{D}$ sounds indicating that the players' finger is approaching a specific object were located too close to each other. Since the game should be equally playable on tablets as well as smartphones, quite some care was needed to make these maps. When the interactable objects had been defined and spread out on the map, the graphical art was made to match the distribution of sound objects. The visual composition is

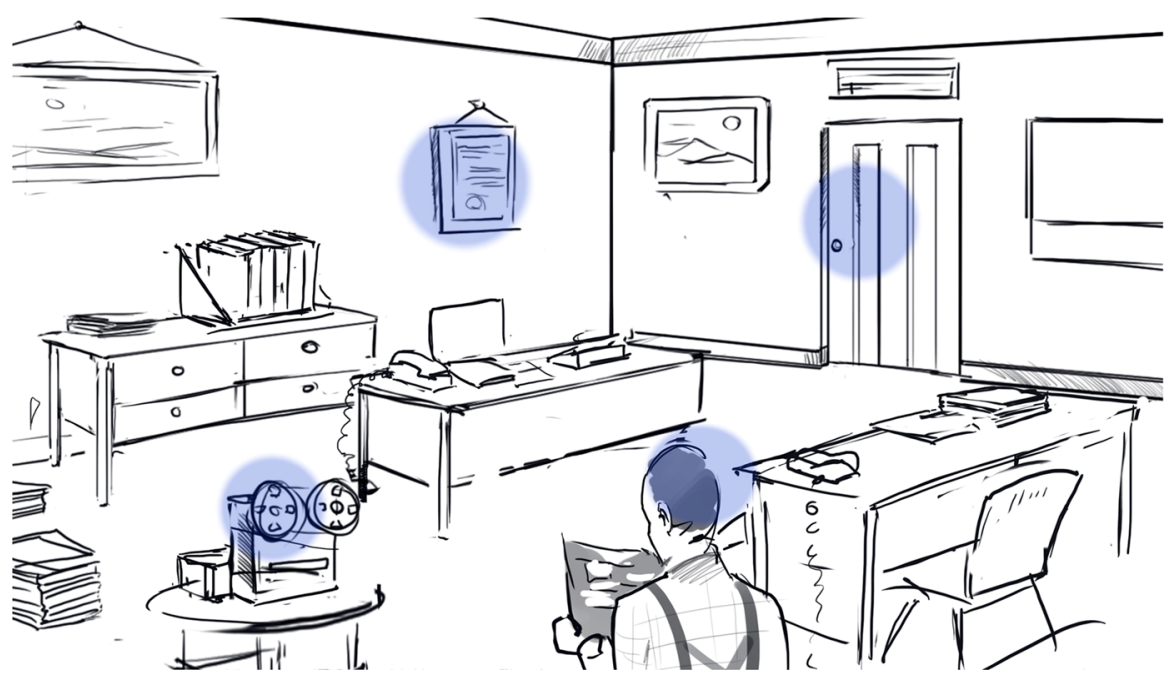

Fig. 2 A concept art image from Frequency Missing showing Karl's office. The blue circles are indicators for where interactable sound objects should be placed on screen (Color figure online) 


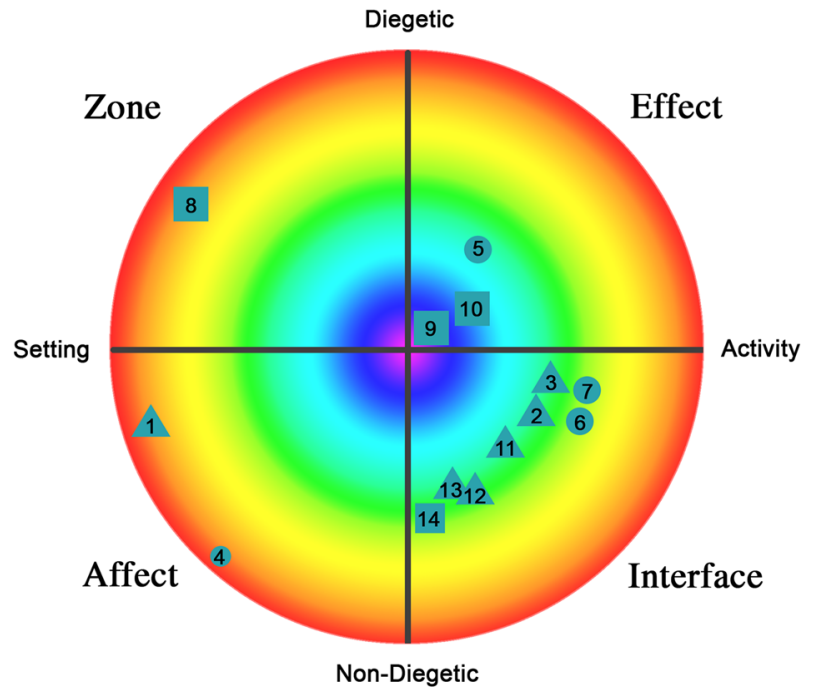

Fig. 3 This is a visualization of the same screen shown in Fig. 2 using the combined model for computer game audio, making it easy to pinpoint the cognitive load on the player. The closer the sounds are to the centre of the circle, the more encoded the sounds are

therefore a result of the distribution of sound objects on the screen, which is quite the opposite of how it is usually construed.

The player interacts with the game by using one finger touching the screen. To make it possible for a visually impaired player and a sighted player to play the same game and share the same experience on as equal terms as possible we needed, in addition to using sound as a game design asset carrying all crucial information, a consistent approach to how the dialogue sequences should be arranged without interfering with the sighted players' experience of the game. An inclusive game design must not discourage groups of players to play the game. The design principles and features that support a visually impaired player must not by any means hinder a sighted player to enjoy the game by flaws in how the game behaves. This ruled out added voice over that tell the player where a dialogue button is located. Instead we let the game as such communicate this information with the aforementioned sound design and every line of language-based information is either integrated as part of the game environment or as part of the game interface.

Buttons on the screen controlling the dialogue always contain written information on what subject the button opens. This information is also spoken by Patricia when holding a finger on the screen at the same height as a button is located vertically. That is, a button's trigger area is always extruded horizontally outside the button making it necessary for the player only to find its position height wise and not its actual visual position on the screen (see Fig. 4). When the player triggers the sound and activates the dialogue button there is also an interface sound. This sound varies in tonality. A dialogue button at the topside of the screen always triggers a high pitched sound and the further down in the structure the player goes, the lower the accompanying interface sound will be. The reason for this is that it maps well with the concept of height and 


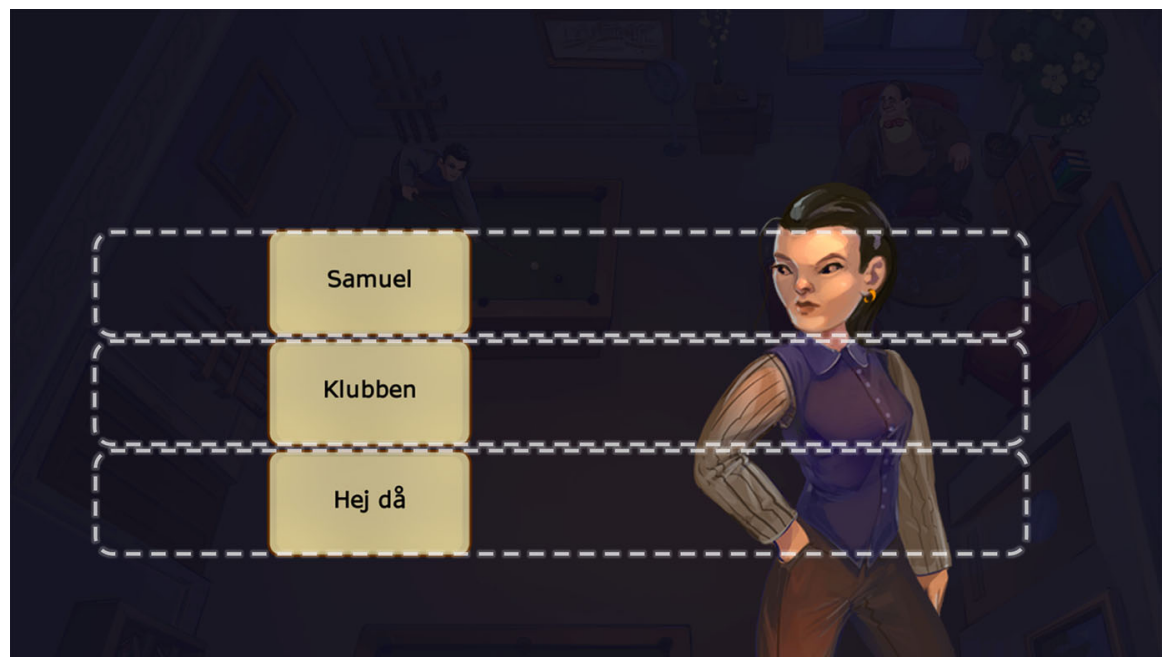

Fig. 4 The dialog interface. The dashed lines indicate the actual interaction area of the buttons

designates the relative positions of the buttons height wise. This design feature does not interfere or disturb a sighted player since it makes use of this natural mapping and the voice over reading the topics of the buttons has a slight delay.

At the beginning of the design process we planned to make use of binaural audio for the sound design. Binaural audio makes it possible to create a very realistic sound experience since the recording technique or a software counterpart mimics the positions of the human ears in the skull. The microphones are commonly placed in a dummy head which provide a so call head shadow for the sound waves. In natural hearing our head affects sound from the right to the left ear for instance and creates interaural time differences (ITDs) as well as interaural level differences (ILD). Instead of using panning between the left and right channel or to locate a sound in either the left or right channel, as stereo recording does, binaural audio works by the virtue of the ITDs and ILDs between the two microphones or virtual ears. The initial tests we made during the first stages of production made us abandon binaural techniques, since at the time of making the game, we could not find a stable enough solution to make for a good listening experience. The motion patterns of the finger on screens were too fast for the binaural sound to playback in a satisfactory manner. Since then there has been a lot of progress in the field of virtual binaural audio for games, and it would be interesting to compare the original game with a version using binaural audio to see if it would improve audio-only players' navigation.

\section{User test}

The game has been evaluated through formal user tests where subjects have been asked to play the first chapter of the game followed by an interview. We have used a qualitative approach for evaluating the subjects' progress in the game and their perception of the story with the aim to find out whether the subjects shared the same 
experience of the narrative in the game. The experiment was conducted according the following procedure:

1. The subject is given a brief introduction to the purpose of the study and information on what data that are collected and that their participation is voluntary and anonymous.

2. The subject is equipped with headphones. After an adjustment of the volume according to the subject's preference, the game is started.

3. The subject plays a short tutorial and then the first chapter of the game. If a subject does not complete this stage within $25 \mathrm{~min}$, the test leader ends the session.

4. The subject answers open questions regarding the experience including aspects related to the story and interaction.

5. The subject answers questions regarding background parameters such as age and experience of gaming.

The gaming device used during the tests was an iPod touch 5G, with a $4^{\prime \prime}$ touch display. The headphones were a pair of AKG 240 Studio. The touch interaction and game states were broadcasted from the iPod using UDP broadcast at 60fps. The device records all finger positions, the active interactable object, and the current stage of the critical path (CP). A laptop computer monitored the broadcasted states. This machine logged all events and visualised them in real time to the test leader. In addition to the logged game interactions all responses from subjects were transcribed directly by the test leader. No audio or video recordings were made. The total time to complete the whole procedure was 50-60 min. As a compensation for participating all subjects were given a $€ 15$ iTunes gift card.

During the play session, the progress of the subject was monitored and if a subject spent three consecutive minutes without making any progress, the test leader provided some hints. The nature of the hints given depended on the type of obstacles a subject experienced. It could be related to the game logic (for example to search for items), the feedback system (for example to listen for interface sounds) or the subject actions (for example to make slower swipes on the touch screen).

The progress subjects made in the game has been evaluated in terms of how far they reached in the Critical Path (CP) shown in Fig. 5. The CP consists of 31 steps of which the first four are tutorial steps before the actual game begins. These 31 steps are the absolute minimum number of interactions that has to be completed to finish the chapter.

The open questions in stage 4 of the test session have been coded in different categories of which perception of setting, characters, and story are the main focuses for this paper.

In total, 19 subjects participated in the test, divided into two main groups: sighted subjects (S-subjects) consisting of five men and five women and visually impaired subjects (VI-subjects) consisting of five men and four women. These groups are not homogeneous. There are for example variations in age (from 15 to 39 years of age) and previous experience of using smartphones and games. From a methodological perspective this is a strength rather than a weakness since our focus was to 
Fig. 5 The objectives of the 31 steps in critical path for the test session

\begin{tabular}{l}
\hline 1. Find Geezer \\
\hline 2. Select dialogue-option "weather" \\
\hline 3. Select "goodbye" \\
\hline 4. Select "new game" \\
\hline 5. Find screw 1 \\
\hline 6. Find screw 2 \\
\hline 7. Find screw 3 \\
\hline 8. Find Karl \\
\hline 9. Select "Richard" \\
\hline 10. Select "Richard vanished" \\
\hline
\end{tabular}

11. Select "enough about Richard"

12. Select "goodbye"

13. Find the door to the lobby

14. Find the door to manager's office
15. Find the manager

16. Select "locked office"

17. Find the key cabinet
15. Find the key cabinet

16. Find the manager

17. Select "locked office"
18. Find the door to the lobby

19. Find the door to the studio

20. Find the electrical switch

21. Select "yes"

22. Find the door to manager's office

23. Find the key cabinet

24. Find the door to the lobby

25. Find the door to Richard's office

26. Find the notice board

27. Find the door to the lobby

28. Find the door to Patricia's office

29. Find Karl

30. Select "yes"

31. Complete puzzle

Legend

- Interactable

- Dialog alternative

- Alternative orders

Special interaction 
qualitatively investigate whether diversified players could share the same gaming experience. Of the VI-subjects the degree of impairment varied: some were blind from birth, some could distinguish light from dark and some could read the text on the screen.

For the scope of this paper, eight open questions from the test are of particular interest:

1. Your spontaneous reactions (to the game)?

2. What is the game about?

3. What is the setting of the game?

4. What are the characteristics of the protagonist of the game?

5. Which characters are there in the game and what roles do they have?

6. What do you think will happen next in the game?

7. What do you think has happened to the colleague who has disappeared?

8. Who do you think is the villain?

These questions all aim to explore to what extent the content of the game has come through to the subjects and if they after playing the first chapter or parts hereof, are able to make assumptions on how the story will unfold and if the subjects enjoyed playing the game. Questions two and three are mainly concerned with the framing story and the location in which it takes place. Questions four and five address the characters in the game and how the players have perceived their characteristics and respective roles in the game. Questions six to eight address to what extent the game's plot elements have been communicated in order for the player to construe a story from them. For example, have the plot elements fired the players' imagination so that they are able to draw speculative conclusions from the various plot elements they have interacted with?

\section{Test results}

\section{Game progress}

In the group of sighted players all but one reached step 31 in the $\mathrm{CP}$ (see Table 1). Among the VI-subjects the result is more diversified, ranging from CP step 13-31. Only four subjects in this group reached step 31 of the $\mathrm{CP}$ during the test session of $25 \mathrm{~min}$.

For S-subjects who finished the chapter, the average time used was $18 \mathrm{~min}$. This was slightly longer for the VI-subjects who used $22 \mathrm{~min}$ on average to finish the chapter. It is notable though, that some VI-subjects completed the game in a shorter time than some S-subjects. The remaining subjects, those that did not reach step 31 in the $\mathrm{CP}$, used all the allotted time. None of the subjects chose to voluntarily end the game session.

Two S-subjects were provided with hints during their game session, according to the 3 min rule presented above. The hints related to problems to locate items in the introductory step (CP 5-6) and problems with the dialog system. Four VI-subjects 
Table 1 The progress of all subjects in terms of the last step reached in the $\mathrm{CP}$ and the total time used

\begin{tabular}{lll}
\hline Subject ID & CP reached & Total playtime (min) \\
\hline S-01 & 31 & 17 \\
S-02 & 31 & 18 \\
S-03 & 31 & 13 \\
S-04 & 31 & 21 \\
S-05 & 30 & 21 \\
S-06 & 31 & 14 \\
S-07 & 31 & 17 \\
S-08 & 31 & 22 \\
S-09 & 31 & 20 \\
S-10 & 31 & 19 \\
VI-01 & 31 & 25 \\
VI-02 & 19 & 25 \\
VI-03 & 13 & 25 \\
VI-04 & 31 & 22 \\
VI-05 & 25 & 25 \\
VI-06 & 31 & 20 \\
VI-07 & 23 & 23 \\
VI-08 & 31 & 21 \\
VI-09 & 18 & 25 \\
\hline
\end{tabular}

were provided with help and for three of those, it related to game logic. The fourth subject experienced problems with the introductory stage and dialog system.

\section{Understanding of the game}

\section{Question 1: Your spontaneous reactions (to the game)?}

Both groups were mainly positive towards the game. Most importantly there were no explicit spontaneous comments from the S-subjects that the inclusive design interfered with the gameplay in a disturbing way. One of the VI-subjects did not like touch screen interfaces in general, since she found those hard to navigate, but was positive towards the game despite this.

Among S-subjects, four of them made comments on the graphics such as "I liked how it looked. It was nice looking", "It was a bit difficult in the beginning to understand that you should drag [your finger] in order to get the circle. I'm an impatient person so I read faster than they talk", "If one didn't look at the screen it was hard to find the doors, hard to find objects with background sounds. It felt like more sounds than I was looking for. The ploink when missing the menu was muddled", and "Didn't notice it was a scrollbar."

All VI-subjects had positive comments about the content of the game and the gaming experience. Two of them wanted clearer instructions concerning how the game interface works. One subject said "First I didn't understand what one should 
do. Where to go. It took a while to understand that one should talk to Karl. The logic of getting into Karl's office was hard to understand." On the other hand, another subject said "Interesting. It was... one immediately understood what one was supposed to do. It wasn't hard." Both these subjects reached CP 31 in less than $22 \mathrm{~min}$.

One subject (CP 31) said "I thought it was a good game. It was clear but just enough. Otherwise I think that other games [for visually impaired players] can be humiliatingly clear."

These spontaneous comments indicate that our design is not overtly or patronizingly supportive in a disturbing manner for either S-subjects or VI-subjects. The players seem to have found the game treating them as players and not impaired players in the case of VI-subjects on the one hand and not sacrificing playability on the other in the case of S-subjects. There is of course a risk that the subjects have been polite and do not really put forth their true opinion of the game. However, the answers on most questions are relatively rich and consistent which indicates that the game design works as intended.

\section{Question 2: What is the game about?}

All subjects in the test identified the main objective of the game, which is to find out what has happened to the protagonist's missing friend and colleague Richard, without any problem. This is one of the first things that are communicated through the plot elements, why also subjects that only reached CP step 13 could identify this objective. The answers are more or less elaborate and the VI-subjects are more verbal and elaborate in their answers. Table 2 shows the number of mentions of specific plot elements of the game and the total amount of mentions for the VIsubjects are 41 whereas the S-subjects only scores 35 .

Table 2 A quantitative comparison between the subjects' identification and recounting of the plot element

\begin{tabular}{llll}
\hline Plot element & Total & S-subj & VI-subj \\
\hline Colleague is missing & 18 & 9 & 9 \\
Objective: find out what happened & 15 & 9 & 6 \\
At radio station & 10 & 3 & 7 \\
Patricia/protagonist & 9 & 3 & 6 \\
Patricia's relationship to the missing colleague & 7 & 4 & 3 \\
Missing since three days & 7 & 4 & 3 \\
Police has closed down the investigation & 5 & 3 & 2 \\
Patricia's first day & 5 & 0 & 5 \\
\hline
\end{tabular}


Question 3: What is the setting of the game?

All subjects except two VI-subjects mention a radio station as one of the settings. One says "media station" and one "in an office space." The latter mentions the radio station setting in the answer to the previous question, so it is not the case that the subject didn't identify the setting but rather misunderstood the question. The location is explicitly introduced at step 8 of the $\mathrm{CP}$ why all subjects in the test have been exposed to it. The cabinet in which Patricia is locked in, is part of a later location, a club, but there are no plot elements revealing this in the chapter played.

\section{Question 4: What are the characteristics of the protagonist of the game?}

There are not any notable differences between the two groups of subjects when they describe the protagonist's core traits. For example, 7 of the S-subjects and 5 of the VI-subjects describe the protagonist as curious. Subjects in both groups also describe her as talkative and stubborn. Most subjects in both groups actually say very few things about her. Notable though is that 3 of the VI-subjects refer to her as out-going, while none of the S-subjects do.

The protagonist is designed to have a core personality that will bias the player to interact with the game environment in a specific way but that also allows for the players to identify with her as their agent in the game.

Question 5: Which characters are there in the game and what roles do they have?

In total there are seven characters that can be identified by name and/or role in the chapter played. There are also a number of non-interactable prop characters in the lobby of the radio station that have an audiovisual presence in the environment but that only serve as a means to make the environment more vivid. Out of the characters that can be identified by name or role, four are audiovisual characters. Karl (sound technician and tech guy, colleague), Monica (the receptionist), Stephanie (news anchor) and the manager. In the chapter, Richard - the missing colleague - is mentioned by name as is duke Silverglans who is said to own a social club. Of course the protagonist Patricia is also part of the first chapter but as noted she is not visually represented but only present by audio and through the player's manipulation of the game environment. The number of identified characters ranged between 4 and 6 for S-subjects and 3 and 6 for VI-subjects. There is in other words only a small difference between the groups, which can be explained by progress in the CP.

The character that has made strongest impression on the subjects is the manager; all subjects mention him as the manager (which is not his name but his role). Some subjects had trouble in remembering the names of the other characters, but manage to provide identifiable characteristics by describing their role in the game (see examples below). The manager does not have a name, only a role, which might explain the high identification score for him. Monica is dominantly referred to as the receptionist, which indicates that her role, just like the manager's, is more 
Table 3 A quantitative comparison between the subjects' identification of the characters

\begin{tabular}{lcccc}
\hline Character & All (by name) & S-Subj & VI-subj & $\begin{array}{l}\text { All (by name or } \\
\text { by identifiable } \\
\text { characteristics) }\end{array}$ \\
\hline Patricia & 4 & 2 & 2 & 9 \\
Richard & 10 & 3 & 7 & 10 \\
Karl & 14 & 7 & 7 & 18 \\
Monica & 4 & 1 & 3 & 17 \\
Manager & 19 & 10 & 9 & 19 \\
Stephanie & 7 & 4 & 3 & 15 \\
Duke Silverglans & 0 & 0 & 0 & 0 \\
\hline
\end{tabular}

prominent than their person. Table 3 presents a summary of the subjects' identification score.

The VI-subjects as a group provide slightly more comments concerning the characters' personalities than the S-subjects. The characters' basic roles in the game are more protruding in the answers from the group of S-subjects. What really comes through in the data is that the efforts put in the character design of the manager worked (Fig. 1 shows his visual appearance). Not only did all subjects identify him as one of the characters, he is also the character that has the most comments regarding his personality. He is deliberately designed as a stereotype of a manager (such as concerned about the image of his enterprise, not too friendly towards his employees, and uninterested). He is also designed to attract the attention of the players as a main suspect behind Richard's disappearance. The VI-subjects generally also provide more comments on his personality than the S-subjects. The roles of the characters are primarily described by all subjects in relation to their subjective construction and interpretation of the story. As an example hereof: one of the S-subject (CP 31) answered "Patricia who was looking for her friend and colleague. Karl in the first room, also colleague. The three felt like they were working in a team. She who was in the lobby, receptionist. Her role I do not know. The manager may know more than he wants to talk about. [...] she was down there who broadcast radio, she was mentor to Patricia." Compared to one of the VIsubjects (CP 31) who answered "On the one hand Patricia who was the main character. Her role was to find Richard and bring the story forward. She had a coworker in the same office called Karl. His role was to be Patricia's backup and supported her. A person named Stephanie who was Patricia's mentor. You were not allowed to meet her so much. Was told that she was cocky and competent. The manager was targeted. Nasty. One person I do not remember her name, she was kind and informative [Monica]. Richard but we don't meet him and one does not get to know much about him. Respected employee who ensured that Patricia got the job." Both these subjects cover the same main topics and the interrelation between the characters and they have a similar experience of playing the game. 
Question 6: What do you think will happen next in the game?

All subjects in the test could speculate on the forthcoming events in the game from their respective position in the $\mathrm{CP}$. The answers vary from speculating on the next chapters to what could happen within the first chapter depending on this. The answers also vary from what they thought would happen very close in time to speculations on how the game as such might end. As in the previous question the answers vary in accordance to how the subjects have construed a story. One of the S-subjects (CP 31) notes that the game environment is built like a big library and hence "Maybe you will find hidden passages behind book shelves. Did not get the feeling that there was something supernatural. More a point click where you have to be a detective without becoming too rebellious towards the manager." None of the VI-subjects have comments about what will happen next in ways similar to this manner. Instead they are rather more to the point in finding clues to solve the mystery. This is probably to some extent an effect of how far they have reached in the $\mathrm{CP}$ but even the VI-subjects that reached step 31 provide less speculative answers than S-subjects.

\section{Question 7: What do you think has happened to the colleague who has disappeared?}

The most common answer to this question is that Richard has been abducted/ kidnapped. There were eight VI-subjects and five or six S-subjects who suggest this (one phrases it as "he has been on to something so someone lured him away", which might be interpreted as someone abducted him). Other suggestions are that he is voluntarily absent (one S-subject), locked up at the radio station by the manager and Stephanie (one S-subject). One VI-subject suggests that he has been murdered. Six of the S-subjects and two of the VI-subjects provide answers that, rather than only addressing the question, suggest why Richard is missing. The most common suggestion is that he has been researching something that might be a threat to someone, that he "knows too much", which is a quite common theme in this genre of narratives (Table 4).

Table 4 A quantitative comparison between the subjects' speculations about what has happened to the missing colleague

\begin{tabular}{llll}
\hline What happened to Richard? & All & S-subj & VI-subj \\
\hline Kidnapped/abducted & $13(14)$ & $5(6)$ & 8 \\
"knows too much" & 5 & 3 & 2 \\
Colleague is involved & 3 & 3 & 0 \\
Locked up & 2 & 1 & 1 \\
Voluntarily absent & 2 & 0 & 2 \\
Blackmail/debt & 1 & 1 & 0 \\
Something with the club & 1 & 1 & 0 \\
Is dead & 1 & 0 & 1 \\
Has published something & 1 & 1 & 0 \\
\hline
\end{tabular}


Table 5 A quantitative comparison between the subjects' speculations of who might be the villain

\begin{tabular}{lcll}
\hline Who is the villain? & Total & S-Subj & VI-subj \\
\hline The manager & 11 & 5 & 6 \\
Karl & 7 & 4 & 3 \\
Monica & 6 & 5 & 1 \\
Not introduced & 6 & 5 & 1 \\
Stephanie & 2 & 2 & 0 \\
Everyone is a suspect! & 2 & 2 & 0 \\
Richard & 1 & 1 & 0 \\
Duke Silverglans & 1 & 1 & 0 \\
Patricia & 0 & 0 & 0 \\
\hline
\end{tabular}

Question 8: Who do you think is the villain?

The game begins (step 5-7 in the CP) with a puzzle sequence in which the protagonist is locked into a cabinet and needs to find a way to get out. This is indicative that someone has actually locked her in. Six S-subjects and two VIsubjects already had begun to speculate who might be a villain, as question 7 indicates. Three S-subjects and one VI-subject think it is someone that is not yet introduced in the game. The manager is the most common suspect but the speculations spans over all characters that are part of the chapter, and even beyond that since there are also speculations that the villain has not yet been present in the game. As noted above (question 5), the manager is the character that is deliberately designed to attract attention from the players. Interesting though, while the VIsubjects provide more extensive stories about what the game is about (question 2), they are notably less inclined to elaborate over who they think is the villain. The S-subjects present double as many possible suspects than the VI-subjects (Table 5).

\section{Conclusions}

\section{Shared experience through a common ground}

From the test we can conclude that the two groups, sighted players and visually impaired players, are able to share the experience of playing the game since they construe the same basic story communicated by the plot elements. Actions and interactable objects in a game do not need to be visual. By using dialogue and narrative voice over as well as clear and well thought out sound design, actions can be defined by audio.

By choosing a game genre which has its primary origin in a long tradition of storytelling, a mystery story, we also chose a common ground between sighted and visually impaired players. The test results indicate that both groups of players have been able to construe similar basic stories, the same setting and have a common idea 
about the personality of the playable character Patricia from the plot elements in the game. They also speculate and put forth similar prognosis concerning what will happen in the next parts of the game. There are differences in how detailed the descriptions are but the core plot elements are recounted by all participants in the test: Richard has disappeared and Patricia wants to find out what has happened to him.

\section{Inclusive game design}

We have also succeeded in designing a game that is inclusive for both groups in that it does not incorporate any obvious distractions to make the game playable and enjoyable for visually impaired players since the strategy we have adopted does not interfere with the gameplay for the sighted player, but is an integral part of the game. The setting of the game at a radio station which is a predominantly audio culture rather than visual culture, as backdrop for the events, makes it possible to integrate audio puzzles as crucial plot elements. From the data gathered in the tests, we can also conclude that the time spent playing the game might pose a problem when sighted and visually impaired players play together. Most sighted players finished the game in shorter time than the visually impaired players in the test. This might pose a problem when a sighted and a visually impaired player plays this kind of game together or at least concurrently, since the sighted player might be impatient towards the slower progress of a visually impaired player. This might be yet an area where an even more inclusive design might be necessary, a design in which different competences complement each other rather than put one competence in favour of another, and in which the time aspect is less of a limitation. For instance, question 2 (see "Understanding of the game" section, above) indicates that visually impaired players listen more carefully to what is said in the dialogue sequences of the game. A game designed for both groups could for example be more focused on cooperation and benefit from the differences between the two groups of players making them more interdependent.

\section{Accessible game culture}

As noted, the use of digital games is widespread and increasing among children, youngsters as well as adults (Entertainment Software Association 2015; Media Council 2013, 2015; Ipsos MediaCT and The Interactive Software Federation of Europe 2012). With our project, we have contributed to the efforts to make the continuously growing game culture and its social dimensions more accessible for visually impaired players. We have found ways of designing video games in a manner that makes them accessible not only for either visually impaired or those that are not but for both these groups. Accessibility is however not the only important issue, but more importantly we have found ways to make a video game joyful to play and emotionally rewarding for both these groups. The design does not frighten sighted players off, and it is not patronizing towards visually impaired players. Both groups can contribute to the act of playing since they share the same basic experience. 


\section{Limitations}

The presented work has some limitations. The study has a focus on a qualitative analysis of open questions. The group of subjects is diverse but limited in number. This is not a problem for a qualitative analysis, but it makes it difficult to draw any definitive conclusions regarding potential differences in how the story is perceived by the different groups. A structured coding scheme could be applied to quantitatively test some of the hypotheses that can be formulated from this study. This will probably require a larger number of participants and may need to extend beyond Sweden. The game is in Swedish, which limits the target group. The group of visually impaired, Swedish speaking subjects without cognitive impairments is very small, which makes it hard to recruit a large number of subjects. It should also be noted that we recruited approximately 15 testers from the VI target group that participated during the development of the game. These persons were disqualified to participate in the research study. An English version of the game would increase the VI target group substantially.

\section{Future work}

We will need to perform more tests to explore whether the game will make it possible for visually impaired players to join a group of sighted players by following the steps that Corsaro (1997) suggests for entering a play situation. That has been beyond the scope of the current study. However, as a first step of designing a game that is enjoyable and comprehensive for sighted and visually impaired players alike, our design has crossed an important threshold; it creates a common ground between these two groups by using the force that lies within narrative as basis for the design. The project has the potential to make digital games even more widespread since we have found ways to design such games in an inclusive manner. In future work, we need to study how games in other genres can be developed inclusively. We anticipate that certain genres will pose larger challenges but that asymmetric game design (Feng et al. 2013) has a big potential for games that include more than one player. If not all players are given the same interface, goals or starting conditions it will be possible to include a more diverse group of players. Luigi's Ghost Mansion (Nintendo 2012) is a good example on how asymmetric game design can be applied.

In addition to visually impaired players, there are more groups that need to be included in gaming culture. For example, we are currently working on extending the presented game to include players with hearing impairment. Although audio has a central role in our game, the most important elements are story and puzzle solving. We believe that these elements can be conveyed in an inclusive way to hearing impaired players as well-without alienating any other group.

Acknowledgement This project has been co-funded by The Swedish Post and Telecom Authority and the University of Skövde. 


\section{Compliance with ethical standards}

Conflict of interest The authors declare that they have no conflict of interest.

Informed consent Informed consent was obtained from all participants.

Open Access This article is distributed under the terms of the Creative Commons Attribution 4.0 International License (http://creativecommons.org/licenses/by/4.0/), which permits unrestricted use, distribution, and reproduction in any medium, provided you give appropriate credit to the original author(s) and the source, provide a link to the Creative Commons license, and indicate if changes were made.

\section{References}

Adams, E. (2010). Fundamentals of game design (2nd ed.). Berkley, CA: New Riders.

Atkinson, M. T., Gucukoglu, S., Machin, C. H. C., \& Lawrence A. E. (2006). Making the mainstream accessible: Redefining the game. In Proceedings of the 2006 ACM SIGGRAPH symposium on videogames, Sandbox'06, New York, NY, USA.

Caillois, R. (1961/2001). Man, play and games. Urbana: Illinois University Press.

Corsaro, W. A. (1997). The sociology of childhood. Thousand Oaks: Pine Forge Press.

CRL. (1976). Colossal cave adventure (Computer game).

Dataspelsbranschen. (2015). Game developer index 2015 (report). Retrieved from http://www. dataspelsbranschen.se/media/152276/gamedeveloperindex\%202015.pdf

Ekman, I. (2007). Sound-based gaming for sighted audiences-experiences from a mobile multiplayer location aware game. In Proceedings of the 2nd audio mostly conference (pp. 148-153). Ilmenau: Fraunhofer Institute for Digital Media Technology.

Embrick, D. G., Wright, T. J., \& Lukacs, A. (Eds.) (2012). Social exclusion, power and video game play: New research in digital media and technology (p. xv, introduction). New York, NY: Lexington Books (Division of Roman and Littlefield).

Entertainment Software Association. (2015). Essential facts about the computer and video game industry (report). Retrieved from http://www.theesa.com/wp-content/uploads/2015/04/ESA-Essential-Facts2015.pdf

Feng, Y., Preston, J. A., \& Skelton, J. (2013). Asymmetric game design and player location: An empirical study on mobile play experiences. In Proceedings of the 18th international conference on computer games. IEEE.

Genette, G. (1983). Narrative discourse. Ithaka, NY: Cornell University Press.

Grammenos, D., Savidis, A., \& Stephanidis, C. (2009). Designing universally accessible games. Computers in Entertainment, 7(1), 8, 1-29.

Huiberts, S. \& van Tol, R. (2008). IEZA: A framework for game audio. Retrieved from http://www. gamasutra.com/view/feature/3509/ieza_a_framework_for_game_audio.php

Infocom. (1980). Zork I: The great underground empire (Computer game).

Ipsos MediaCT \& Interactive Software Federation of Europe. (2012). Videogames in Europe: European summary report (report). Retrieved from http://www.isfe.eu/sites/isfe.eu/files/attachments/euro_ summary_-_isfe_consumer_study.pdf

Lucas Arts. (1990). The secret of monkey Island (Computer game).

Lucas Arts. (1993a). Day of the tentacle (Computer game).

Lucas Arts. (1993b). Sam \& Max hit the road (Computer game).

Murch, W. (1998). Dense clarity—Clear density. Retrieved from http://www.ps1.org/cut/volume/murch. html

Nintendo. (2012). Luigi's ghost mansion (Computer game).

Oren, M. A. (2007). Speed sonic across the span: Building a platform audio game. In CHI'07 extended abstracts on human factors in computing systems. New York: ACM.

Söderqvist Dunkers, T. (2011). Talande möten mellan ungdomar: Villkor för delaktighet i kamratgemenskap - ungdomar med synnedsättning berättar. Master thesis, Stockholm: Dept. for Pedagogy and Didactics, University of Stockholm (English title: Talking encounters: What do young people with severe visual impairment say about terms of participation in the peer culture?). 
Somethin' Else. (2010). Papa Sangre (Mobile game).

Somethin' Else. (2012). Papa Sangre II (Mobile game).

Stukat, K.-G. (2002). Three decades of integrated education. In S. Hegarty \& M. Alur (Eds.), Education \& children with special needs-From segregation to inclusion (pp. 173-180). New Dehli: Tejeshwar Sing for Sage Publications India Pvt Ltd.

Swedish Media Council. (2013). Kids \& media 2012/13 (report). (Statens Medieråd, Ungar \& Medier 2012/13). Retrieved from http://www.statensmedierad.se/download/18.3d35018c150001239d91a 877/1443358522280/Ungar_och_medier_2013_fullfarg.pdf. English summary: http://www. statensmedierad.se/download/18.1957a5a61500017241926d9e/1452244272247/Kids-andmedia-2013.pdf

Swedish Media Council. (2015). Kids \& media 2015 (report) (Statens Medieråd, Ungar \& Medier 2015). Retrieved from http://www.statensmedierad.se/download/18.7a953dba14fef1148cf3b32/14428419 39189/Ungar-och-medier-2015.pdf

Synskadades riksförbund. (2016a). Vem är synskadad. Retrieved from http://www.srf.nu/leva-medsynnedsattning/om-synskador/vem-ar-synskadad/

Synskadades riksförbund. (2016b). Skolor och undervisning. Retrieved from http://www.srf.nu/om-oss/ museet/synskadades-historia/skolor-och-undervisning/

Wilhelmsson, U., \& Wallén, J. (2011). A combined model for the structuring of computer game audio. In M. Grimshaw (Ed.), Game sound technology and player interaction: Concepts and developments (pp. 98-132). Hershey: IGI Global.

UIf Wilhelmsson is a senior lecturer at the University of Skövde. His research interests include computer game audio, story driven game design and game development.

Henrik Engström is a senior lecturer at the University of Skövde. His research interests include game development, serious games and game design.

Jenny Brusk is a senior lecturer at the University of Skövde. Her research interests include game dialogue systems, game design, and inclusive game development.

Per Anders Östblad is a lecturer at the University of Skövde. His area of interests include game audio, game accessibility and game audio implementation. 